\title{
History effects on the gas exchange between a bubble and a liquid
}

\author{
Shigan $\mathrm{Chu}^{*}$ and Andrea Prosperetti ${ }^{\dagger}$ \\ Department of Mechanical Engineering, Johns Hopkins University, Baltimore, Maryland 21218, USA
}

(Received 17 May 2016; published 17 October 2016)

\begin{abstract}
Diffusive processes exhibit a strong dependence on history effects. For a gas bubble at rest in a liquid, such effects arise when the concentration of dissolved gas at the bubble surface, dictated by Henry's law, depends on time. In this paper we consider several such situations. An oscillating ambient pressure field causes the occurrence of rectified diffusion of gas into or out of the bubble. Unlike previous investigators, who considered the opposite limit, we study this process for conditions when the diffusion length is larger than the bubble radius. It is found that history effects are important in determining the threshold conditions. Under a static ambient pressure, the time dependence of the gas concentration can arise due to the action of surface tension, which increases the gas pressure as the bubble dissolves or, when the bubble contains a mixture of two or more gases, due to the different rates at which they dissolve. In these latter cases history effects prove mostly negligible for bubbles larger than a few hundred nanometers.
\end{abstract}

DOI: 10.1103/PhysRevFluids.1.064202

\section{INTRODUCTION}

Dependence on past history is one of the distinctive features of diffusive processes. In this paper we study the consequences of this dependence on the mass exchange between a gas bubble and the ambient liquid super- or undersaturated with dissolved gas. The driver for the exchange is the difference between the concentration of the diffusing species at the surface of the bubble and in the surrounding liquid. The former is dictated by the bubble internal pressure according to Henry's law. We consider the limit of small radial velocities, which enables us to omit the effects of convection, focusing specifically on diffusion and the attendant history phenomena. We first develop a general theory (Sec. II) followed by some general scaling considerations (Sec. III), and then illustrate its consequences for cases in which the ambient pressure oscillates (Sec. IV) or is constant (Secs. V and VI), finding strong effects in the former situation and small ones in the latter two.

The mass transfer between a bubble and the surrounding liquid in an oscillating pressure field is well known in the acoustics literature, where it is referred to as rectified diffusion (see, e.g., Refs. [1-3]). The adjective rectified refers to the fact that a net inflow of gas dissolved in the liquid becomes possible in certain conditions. This phenomenon is due to the alternating expansion and contraction of the bubble, which causes the pressure of the gas contained in it to oscillate. Due to Henry's law, the concentration of dissolved gas in the liquid at the bubble surface also oscillates and, if it becomes sufficiently small during expansion, the resulting diffusive influx may be large enough to balance the mass lost during contraction when the pressure rises.

A very complete theory of rectified diffusion has been developed by Fyrillas and Szeri [4] for conditions in which the diffusion layer, of order $\sqrt{D / \omega}$ (with $D$ being the gas diffusivity in the liquid and $\omega$ being the angular frequency of the ambient pressure field), is much thinner than the bubble radius. In this limit, the alternating thinning and thickening of the diffusive boundary layer at the bubble surface play a dominant role in determining the mass transfer. Here we focus on the opposite

\footnotetext{
*schu13@jhu.edu

${ }^{\dagger}$ Present address: Department of Mechanical Engineering, University of Houston, Houston, TX 77204, USA; also Faculty of Science and Technology, Impact Institute and J. M. Burgers Center for Fluid Dynamics, University of Twente, 7500AE Enschede, The Netherlands; prosperetti@jhu.edu
} 
limit, in which the frequency is low and the diffusion layer is much thicker than the bubble radius. A situation of this type might be encountered, for example, in the case of bubbles present in a liquid, the container for which is subject to mechanical vibrations; examples of other possible occurrences are low-frequency acoustic fields or bubbles in a turbulent liquid flow. Under these conditions there is no diffusive boundary layer and rectified diffusion is only dependent on the difference in the surface area of the bubble upon expansion and contraction.

The bubble-liquid mass exchange in a constant pressure field was first studied in a by-now classic paper by Epstein and Plesset [5], who treated the problem of the dissolution or growth of a gas bubble in a liquid under- or supersaturated with gas. More recently their approach has been extended to the dissolution or growth of single-component drops in an immiscible liquid $[6,7]$ and to the study of nanodrops and nanobubbles [8]. As long as surface tension and dynamical effects are negligible, whatever the bubble radius, the gas pressure in the bubble balances the constant ambient pressure and the dissolved gas concentration at the bubble surface remains constant, according to Henry's law. With surface tension, however, the bubble internal pressure comes also to depend on the bubble radius and, therefore, it changes as the bubble grows or dissolves.

Epstein and Plesset [5] did not explicitly account for the time dependence of this effect, assuming an instantaneous adjustment of the dissolved gas concentration to the changing boundary condition and, in so doing, they omitted history effects. This situation is considered explicitly in Sec. V, with the conclusion that their procedure is essentially justified as long as the bubble is larger than a few hundred nanometers. Another situation in which the dissolved gas concentration at the bubble surface depends on time, considered in Sec. VI, is that of a multicomponent bubble, the constituents of which dissolve and diffuse at different rates. Here again history effects prove of relatively minor importance.

History effects have also been studied in a very recent paper [9] focusing, however, on situations different from those considered here, namely the response of the bubble when the ambient pressure undergoes jump discontinuities and rectified diffusion in the limit of small-amplitude oscillations. The method of investigation is similar, except for the use of a modified time variable (a point which we briefly consider in the appendix). This paper also contains several interesting observations on the nature of the approximations made in the mathematical model.

The phenomena studied in the present work are similar to those considered in a previous recent paper [10] devoted to the dissolution or growth of a multicomponent drop. In that case, the time dependence of the surface concentration of the diffusing species is a consequence of their mutual interaction as the composition of the drop varies in time.

\section{ANALYSIS}

We follow the formulation of Epstein and Plesset [5], according to which a bubble, containing an incondensible gas at a pressure $p_{g}$, is instantaneously exposed to an infinite liquid medium containing the same gas dissolved with a uniform concentration $\rho_{g}^{i}$. The ambient pressure in the liquid is $p_{\infty}$ and diffusion and pressure variation are so slow that mechanical equilibrium may be assumed at the bubble surface, according to which

$$
p_{g}=p_{\infty}+\frac{2 \sigma}{R},
$$

where $\sigma$ is the surface tension coefficient and $R$ is the bubble radius. As in Ref. [5] we assume that the bubble remains spherical and that it does not move relative to the host liquid.

The liquid concentration $\rho_{g}^{s f}$ of the dissolved gas at the bubble surface is determined by Henry's law as

$$
\rho_{g}^{s f}=K(T) p_{g}
$$

in which $K(T)$ is a function of the absolute temperature $T$. The difference $\rho_{g}^{s f}-\rho_{g}^{i}$ sets up a diffusive flux $-D \nabla \rho_{g}$ which causes inflow or outflow of gas from the bubble; $D$ is the mass diffusivity of 
the gas in the liquid. In order to characterize the solubility of the gas it is useful to follow Ref. [5], introducing a parameter

$$
d=\frac{\rho_{\mathrm{sat}}}{\rho_{b}^{0}}=\frac{K(T) R_{G} T}{M_{g}},
$$

with $\rho_{\text {sat }}$ being the density of gas dissolved in the liquid at saturation under the pressure $p_{\infty}^{0}, \rho_{b}^{0}$ being the density of the free gas at the same pressure $p_{\infty}^{0}, R_{G}$ being the universal gas constant, $T$ being the absolute temperature, and $M_{g}$ being the molecular mass of the gas.

For a bubble undergoing spherically symmetric motion, the convection-diffusion equation for the density $\rho_{g}$ of gas dissolved in the liquid may be written as

$$
\frac{\partial \rho_{g}}{\partial t}=\frac{1}{r^{2}} \frac{\partial}{\partial r}\left[D r^{2} \frac{\partial \rho_{g}}{\partial r}-R^{2} \dot{R}\left(\rho_{g}-\rho_{g}^{i}\right)\right],
$$

in which $R=R(t)$ is the bubble radius, $\dot{R}=d R / d t$, and $r$ is the distance from the bubble center. For motions taking place on a time scale $\omega^{-1}$ sufficiently long that the diffusion length $\sqrt{\pi D / \omega}$ exceeds the radius, the characteristic length for the gradient is the bubble radius itself and we have the estimate

$$
\frac{R^{2} \dot{R}\left(\rho_{g}-\rho_{g}^{i}\right)}{D r^{2} \partial \rho_{g} / \partial r} \sim \frac{R^{2}}{r^{2}} \frac{R \dot{R}}{D},
$$

in which the second fraction may be considered a Péclet number. Thus, the last term in (4) will be negligible compared with the diffusion term provided the Péclet number is small, which we assume. In an oscillating pressure field, if the bubble radius changes by $\Delta R$ during the time $\omega^{-1}$, $\dot{R} \sim \omega \Delta R$ and $R \dot{R} / D \sim\left(R^{2} \omega / D\right)(\Delta R / R)$. The first factor is the square of the ratio of the radius to the diffusion length and is essentially the square of the parameter $\Lambda$ introduced later in (22).

On the basis of these considerations and assumptions we set $u=r\left(\rho_{g}-\rho_{g}^{i}\right)$, drop the convective term and recover the simpler diffusion equation

$$
\frac{\partial u}{\partial t}=D \frac{\partial^{2} u}{\partial r^{2}}
$$

which will be used in this paper. This is the standard approximation made in a majority of papers on the present subject, most recently in Ref. [9]. Some further considerations on these issues will be found in the next section. The boundary conditions are

$$
\rho_{g}[R(t), t]=H(t) \rho_{g}^{s f}(t), \quad \lim _{r \rightarrow \infty} \rho_{g}=\rho_{g}^{i},
$$

where the Heaviside function $H(t)$ enforces the fact that the boundary condition is applied instantaneously at $t=0+$, the concentration being $\rho_{g}^{i}$ at earlier times. The initial condition is

$$
\rho_{g}(r, 0)=\rho_{g}^{i}
$$

The rate at which $m_{g}$, the mass of gas contained in the bubble, varies is given by

$$
\frac{d m_{g}}{d t}=\left.4 \pi R^{2}(t) D \frac{\partial \rho_{g}}{\partial r}\right|_{r=R(t)} .
$$

Unlike Epstein and Plesset, we allow here $p_{\infty}$ to be time dependent but to vary so slowly that the quasiequilibrium condition (1) is very nearly satisfied at every instant. The condition for the validity of this approximation is that the frequency be much smaller than the bubble resonance frequency, of the order of $\sqrt{3 p_{\infty} /\left(\rho R^{2}\right)}$. Ordinarily this condition is much less stringent than the previous one related to the neglect of convective terms. 
In response to the changing ambient pressure, the bubble radius will also change. If the gas in the bubble can be described by the ideal gas law, the bubble radius satisfies the relation

$$
p_{g}\left(\frac{4}{3} \pi R^{3}\right)=\frac{m_{g}}{M_{g}} R_{G} T,
$$

in which $p_{g}$ is given by (1). In view of the assumed slow variation of the pressure, the gas will behave essentially isothermally so that $T$ can be considered to be a constant.

In principle, with a significant amount of vapor in the bubble, several new effects become important: The mutual diffusion of the gas and vapor, latent heat, and temperature variations both in the gas-vapor mixture and in the liquid. As the focus of this paper is to study the effect of the time dependence of the dissolved gas concentration at the bubble surface, we disregard these complications here simply noting that a constant vapor pressure contribution can be simply added to the left-hand side of (1) if necessary.

Following the work of Epstein and Plesset and many other investigators [11-13], in the solution of the mathematical problem posed by (6), (7), and (8) we will treat $R$ as a parameter. Tao [14,15] tried to improve on this approximation but his method of solution leads to an infinite series that must be truncated, introducing errors that are difficult to quantify. A better approach was developed in a recent paper [9] by the introduction of a modified time variable. The radius of a dissolving bubble decreases very slowly for a significant fraction of its lifetime; it is only near the end of the process that it varies rapidly. In this case, as shown in the appendix, the difference with our procedure is small. In the case of an oscillating pressure field, the error is of the order of $\Delta R / R$, and therefore it is of the same order as that introduced by the neglect of convection, as explained in the next section. Hence, treating the radius in a parametric way is consistent with the other approximations made in this study.

We take the Laplace transform (denoted by a tilde) and solve the resulting differential equation to find

$$
\tilde{u}(r, s)=\left.\tilde{u}\right|_{r=R} e^{-\sqrt{s / D}(r-R)} .
$$

In terms of $\tilde{u}$ we have

$$
\left.\frac{\partial \tilde{\rho}}{\partial r}\right|_{r=R}=-\left[\frac{\tilde{u}(R, s)}{R^{2}}+\frac{1}{R} \frac{s \tilde{u}(r, s)}{\sqrt{D s}}\right]_{r=R},
$$

which can be readily inverted. When the result is inserted into (9) we find

$$
\frac{d m_{g}}{d t}=4 \pi R^{2}(t) D\left[\frac{\rho_{g}^{i}-\rho_{g}^{s f}(0)}{\sqrt{\pi D t}}+\frac{\rho_{g}^{i}-\rho_{g}^{s f}(t)}{R}-\int_{0}^{t} \frac{d \rho_{g}^{s f}(\tau)}{d \tau} \frac{d \tau}{\sqrt{\pi D(t-\tau)}}\right] .
$$

The first term in the right-hand side is due to the discontinuity of the boundary condition at $t=0$. If $\rho_{g}^{s f}$ is independent of time, this result coincides with that of Ref. [5]. The last term is the history term due to the variation in time of the surface concentration, which is a consequence of the time dependence of the bubble pressure caused by the variation of the ambient pressure, surface tension, or other factors. The difference with Ref. [5] is even more evident upon rewriting this equation identically as

$$
\frac{d m_{g}}{d t}=4 \pi R^{2}(t) D\left\{\left[\rho_{g}^{i}-\rho_{g}^{s f}(t)\right]\left(\frac{1}{\sqrt{\pi D t}}+\frac{1}{R}\right)-\int_{0}^{t} \frac{d \rho_{g}^{s f}(\tau)}{d \tau}\left[\frac{1}{\sqrt{\pi D(t-\tau)}}-\frac{1}{\sqrt{\pi D t}}\right] d \tau\right\} .
$$

The first term in the right-hand side is the only term occurring in the original theory of Ref. [5].

After a discussion of orders of magnitude in the next section, we illustrate the effect of the new memory term in (13) or (14) for the case in which the time dependence of $\rho_{g}^{s f}$ is due to the time 
dependence of the ambient pressure or, in a constant pressure field, to the effect of surface tension or to the presence of different gases.

\section{SCALING}

It was shown in the previous section that the convective term of the diffusion equation is small compared with the diffusion term provided the Péclet number is small. We now turn to a consideration of the other term in the convective derivative, namely $\partial \rho_{g} / \partial t$. Proceeding as for the convective term, the ratio of the two terms in the convective derivative can be estimated as

$$
\frac{\left(R^{2} / r^{2}\right) \dot{R} \partial \rho_{g} / \partial r}{\partial \rho_{g} / \partial t} \sim \frac{\dot{R} t_{\text {diff }}}{R},
$$

in which $t_{\text {diff }}$ is the characteristic time scale over which $\rho_{g}$ varies appreciably.

In a static pressure field $t_{\text {diff }}$ can be estimated as $R^{2} / D$, while $\dot{R}$ will be of the order of the initial radius $R_{0}$ divided by the total dissolution time $t_{\text {diss }}$ which, for a monocomponent bubble, is of the order of $R_{0}^{2} /\left[d D\left(1-\rho_{g}^{i} / \rho_{\text {sat }}\right)\right]$, according to Epstein and Plesset. In this way we find

$$
\frac{\left(R^{2} / r^{2}\right) \dot{R} \partial \rho_{g} / \partial r}{\partial \rho_{g} / \partial t} \sim\left(1-\frac{\rho_{g}^{i}}{\rho_{\mathrm{sat}}}\right) d,
$$

a result previously given in Ref. [16]. In this case, therefore, it is consistent to neglect the convective term provided $d$ is small or the liquid is nearly saturated with gas.

The situation is different in an oscillating pressure field with frequency $\omega$ because, in this case, the previous estimate of $\dot{R}$ is only applicable provided $\omega t_{\text {diss }}$ is less than 1, i.e., at very low frequencies. When $\omega t_{\text {diss }}>1$, the proper time scale for both $\dot{R}$ and $\partial \rho_{g} / \partial t$ is just $1 / \omega$ and the ratio of the two terms is of the order

$$
\frac{\left(R^{2} / r^{2}\right) \dot{R} \partial \rho_{g} / \partial r}{\partial \rho_{g} / \partial t} \sim \frac{\dot{R} / R}{\omega} \simeq \frac{\Delta R}{R},
$$

with $\Delta R$ being of the order of the oscillation amplitude of the radius. When the radius changes are small, the convective term is of higher order and can be neglected, which justifies the linearization of the diffusion equation. In this case, the boundary condition at the moving bubble surface can be treated as a domain perturbation as shown in one of the cases treated in Ref. [9]. When $\Delta R \sim R$, however, the two terms of the convective time derivative are of a comparable order and, therefore, the ratio of $\partial \rho_{g} / \partial t$ to diffusion is also of the order of the Péclet number. In these conditions, when this number is small, it would be consistent to just use the steady-state diffusion equation treating time simply as a parameter. In this quasisteady approximation, the only surviving term in the equation (13) for $d m_{g} / d t$ would then be the middle one, proportional to $1 / R$, and all effects explicitly dependent on time, including history, will be of the order of the Péclet number and, hence, small. When $\Delta R / R$ is not small, omission of the convective term in spite of the retention of $\partial \rho_{g} / \partial t$ is unjustified from a purely mathematical viewpoint. A pragmatic justification for our procedure is that it enables us to explicitly identify the presence of history effects and to estimate their magnitude. Furthermore, as will be shown in the next section in connection with Fig. 7, the effect of the magnitude of the ratio $\Delta R / R$ is rather small, which lends further justification to the simplification adopted in this paper.

\section{MASS TRANSFER IN AN OSCILLATING AMBIENT PRESSURE}

Bubble growth (or delayed dissolution) by rectified diffusion is a fundamentally nonlinear processes. First, for equal positive and negative ambient pressure changes, the bubble radius increases more upon expansion than it decreases upon contraction due the nonlinearity of the pressure-volume relation. (A contributing factor is the nonlinearity of the Rayleigh-Plesset equation equation governing the radial motion, which is neglected here.) Thus, the surface area when the 
bubble expands and absorbs gas from the surroundings is larger than when it contracts and loses gas. Second, upon expansion, the diffusion boundary layer at the bubble surface is stretched and thins, which increases the inward-directed mass flux while, when it contracts, the boundary layer thickens and the outward-directed mass flux decreases. In the limit considered here this second process, as well as inertia, is negligible and the phenomenon rests solely on the difference in the surface areas upon expansion and contraction of the bubble.

We focus on the threshold conditions separating bubble growth from dissolution and consider sinusoidal ambient pressure oscillations writing

$$
p_{\infty}(t)=p_{\infty}^{0}\left(1-P_{*} \sin \omega t\right)
$$

with $P_{*}$ being the dimensionless pressure amplitude. A length $R_{e}$ useful to characterize the bubble size can be defined by

$$
\frac{4}{3} \pi R_{e}^{3} p_{\infty}^{0}=\frac{\left\langle m_{g}\right\rangle}{M_{g}} R_{G} T
$$

in which $\left\langle m_{g}\right\rangle$ is the average mass of gas in the bubble, which is a constant in steady threshold conditions. We set

$$
t_{*}=\omega t, \quad R_{*}=\frac{R(t)}{R_{e}}, \quad p_{\infty}^{*}\left(t_{*}\right)=\frac{p_{\infty}(t)}{p_{\infty}^{0}}, \quad \rho_{*}^{s f}=\frac{\rho_{g}^{s f}}{\rho_{\mathrm{sat}}},
$$

with $\rho_{\text {sat }}=K(T) p_{\infty}^{0}$. In dimensionless form the mass transfer equation (13) is

$$
\frac{d m_{g *}}{d t_{*}}=\frac{3}{\pi} d \frac{\sqrt{\pi D / \omega}}{R_{e}} R_{*}^{2}\left[\frac{\rho_{g *}^{i}-\rho_{g *}^{s f}(0)}{\sqrt{t_{*}}}+\frac{\sqrt{\pi D / \omega}}{R_{e}} \frac{\rho_{g *}^{i}-\rho_{g *}^{s f}(t)}{R_{*}}-\int_{0}^{t_{*}} \frac{d \rho_{g *}^{s f}\left(\tau_{*}\right)}{d \tau_{*}} \frac{d \tau_{*}}{\sqrt{t_{*}-\tau_{*}}}\right],
$$

in which $m_{g *}=m_{g} /\left\langle m_{g}\right\rangle$ and $d$ is the solubility parameter defined earlier in (3). An important parameter appearing in this equation is

$$
\Lambda=\frac{R_{e}}{\sqrt{\pi D / \omega}}
$$

which is the ratio of of the average bubble radius to the diffusion penetration length. The bubble internal pressure is nondimensionalized in the same way:

$$
p_{g}^{*}=\frac{p_{g}}{p_{\infty}^{0}}=p_{\infty}^{*}+\frac{\alpha}{R_{*}}=p_{\infty}^{*}+\frac{\beta}{\Lambda R_{*}}
$$

where

$$
\alpha=\frac{2 \sigma}{p_{0}^{\infty} R_{e}}, \quad \beta=\frac{2 \sigma}{p_{0}^{\infty} \sqrt{\pi D / \omega}} .
$$

We thus see that the problem contains five parameters, namely $\rho_{g *}^{i}, \Lambda, P_{*}, d$, and $\alpha$ (or $\beta$ ). At threshold conditions, any one of them will be a function of the remaining four.

Threshold conditions for rectified diffusion are identified by the requirement that no net mass transfer occurs over a complete cycle of pressure oscillations:

$$
\int_{t_{*}}^{t_{*}+2 \pi / \omega} R_{*}^{2}\left[\frac{\sqrt{\pi D / \omega}}{R_{e}} \frac{\rho_{g *}^{i}-\rho_{g *}^{s f}(t)}{R_{*}}-\int_{0}^{t_{*}} \frac{d \rho_{g *}^{s f}\left(\tau_{*}\right)}{d \tau_{*}} \frac{d \tau_{*}}{\sqrt{t_{*}-\tau_{*}}}\right] d t=0 .
$$

Here we have omitted the transient term proportional to $t^{-1 / 2}$ in (21), the effect of which decreases with time. 


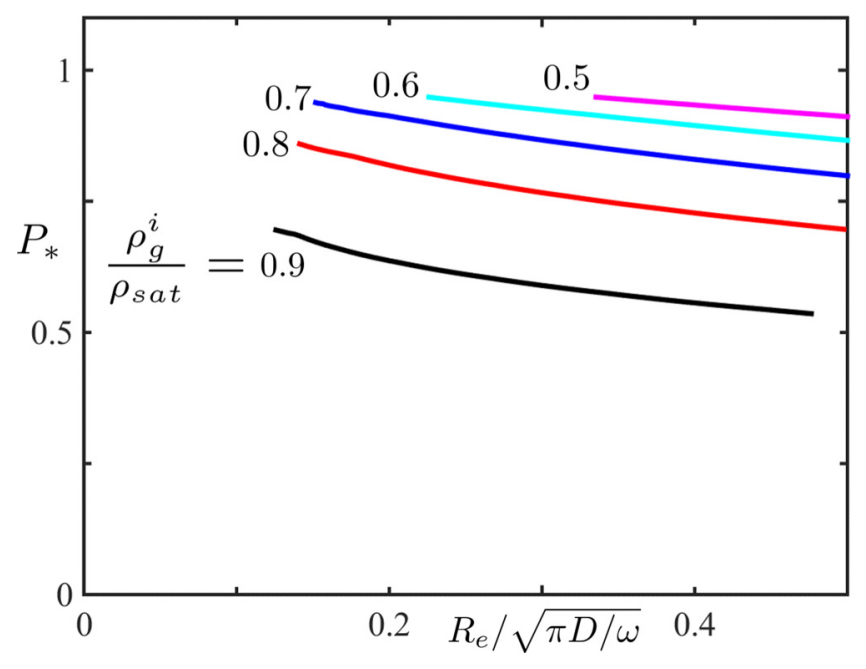

FIG. 1. Dimensionless rectified diffusion threshold amplitude $P_{*}$ vs dimensionless bubble radius $R_{e} / \sqrt{\pi D / \omega}$ for different values of the liquid undersaturation $\rho_{g}^{i} / \rho_{\text {sat }}$, surface tension effects not included.

Before turning to the results it is useful to note that, as a consequence of the equation of state,

$$
\frac{\left[1-P_{*} \sin t_{*}+\alpha / R_{*}\left(t_{*}\right)\right] R_{*}^{3}\left(t_{*}\right)}{1+\alpha}=\frac{m_{g}(t)}{\left\langle m_{g}\right\rangle} .
$$

According to this relation, the bubble radius responds to both the external pressure and changes in the bubble contents. Consider the beginning of a pressure cycle in which the pressure falls. Until the pressure inside the bubble becomes close to the saturation value corresponding to the gas content of the liquid, gas keeps diffusing out of the bubble and the radius expands less than it would in the absence of diffusion. When the ambient pressure is low enough, gas starts diffusing into the bubble and the radius starts expanding more than it would without diffusion. This expansion period which, due to the area effect mentioned before, is crucial for rectified diffusion, becomes longer as the pressure amplitude is increased. When the ambient pressure starts rising, the increased mass of gas in the bubble limits the bubble compression and, therefore, decreases the mass loss. Since these effects depend on diffusion, which is a slow process, one may expect their influence to decrease as frequency is increased. These considerations help to interpret the results that we now describe. For definiteness we present numerical results for the case of air and water at $22{ }^{\circ} \mathrm{C}$, for which $d=0.02$.

We begin by neglecting surface tension effects so that, with $d$ fixed, the free parameters reduce to 3 , namely the water super- or undersaturation $\rho_{g}^{i} / \rho_{\text {sat }}$, the dimensionless radius $R_{e} / \sqrt{\pi D / \omega}$, and the dimensionless pressure amplitude $P_{*}$. Figure 1 is a graph of the threshold amplitude $P_{*}$ vs the parameter $R_{e} / \sqrt{\pi D / \omega}$ for different values of the undersaturation $\rho_{g}^{i} / \rho_{\text {sat }}$. It is clear from (26) that, in the absence of surface tension, the radial oscillations become larger as $P_{*}$ approaches 1 . This circumstance makes a precise determination of the threshold numerically delicate in this range and, for this reason, our exploration of the parameter $P_{*}$ does not extend all the way to 1 . This difficulty becomes more acute with increasing $\rho_{g}^{i}$ as then the amplitude of the mass fluxes also becomes very large. The range of the horizontal axis is limited by the requirement that $R_{e}$ be smaller than $\sqrt{\pi D / \omega}$ for the present approximations to be justified, as discussed in Sec. II.

The results of Fig. 1 conform with the analysis provided before in connection with (26). The threshold amplitude increases with increasing undersaturation (i.e., decreasing gas content in the liquid) and also increases as the frequency decreases due to the increasing importance of diffusive effects. 


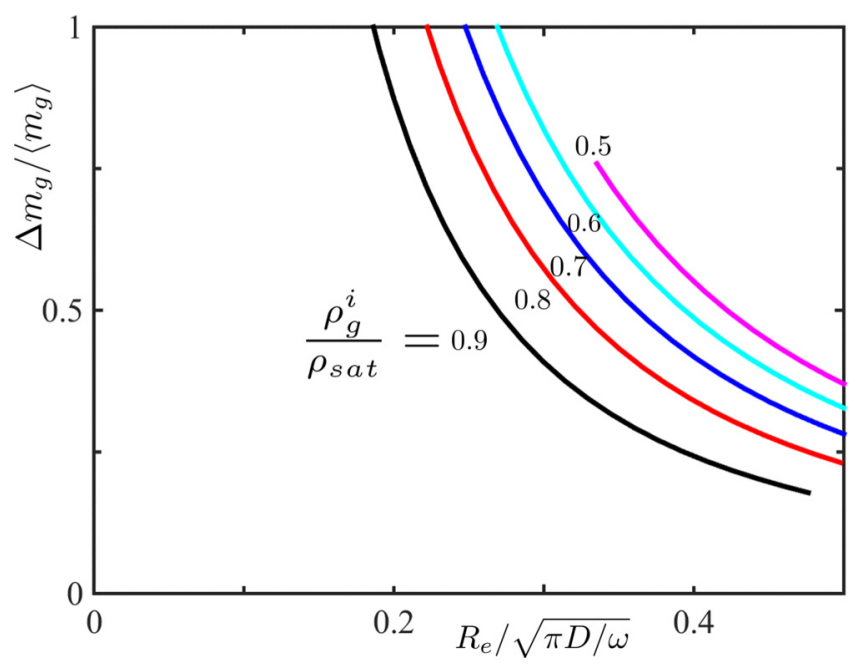

FIG. 2. Dimensionless oscillation amplitude of the mass of gas in the bubble vs the dimensionless bubble radius $R_{e} / \sqrt{\pi D / \omega}$ for different liquid undersaturations as in Fig. 1, surface tension effects are not included.

Additional insight into these results is provided by Figs. 2 and 3. The former shows the amplitude of the mass oscillations $\Delta m_{g}=m_{g}$, max $-m_{g}$, min normalized by $\left\langle m_{g}\right\rangle$, while the latter is the nondimensional radius excursion, $\Delta R / R_{e}$, with $\Delta R=R_{\max }-R_{\min }$, both as functions of the parameter $R_{e} / \sqrt{\pi D / \omega}$. Each line corresponds to one value of $\rho_{g}^{i} / \rho_{\text {sat }}$. As expected, the mass amplitude rapidly increases as the frequency is decreased, reaching $100 \%$ of the mean value in the parameter range covered by the figure. Balancing the increasing mass losses taking place at low frequency requires an increased mass inflow, which in turns requires a large area increase and a significant radial amplitude. Conversely, as the frequency increases, mass losses are reduced, the radial amplitude becomes more tightly connected with the ambient pressure oscillations, and the radial oscillation amplitude decreases.

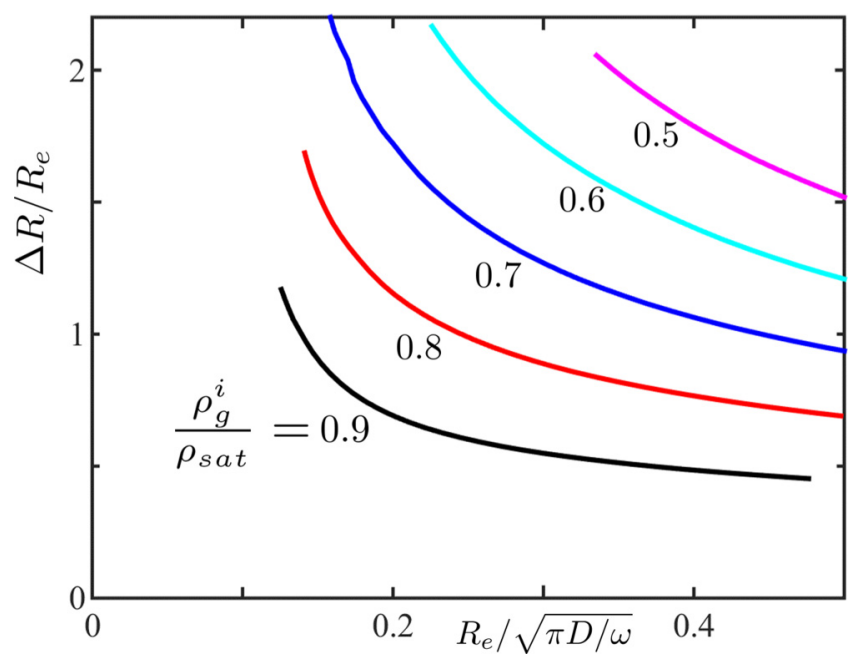

FIG. 3. Dimensionless oscillation amplitude of the bubble radius vs the dimensionless bubble radius $R_{e} / \sqrt{\pi D / \omega}$ for different liquid undersaturations as in Fig. 1, surface tension effects are not included. 


\section{HISTORY EFFECTS ON THE GAS EXCHANGE BETWEEN A ...}

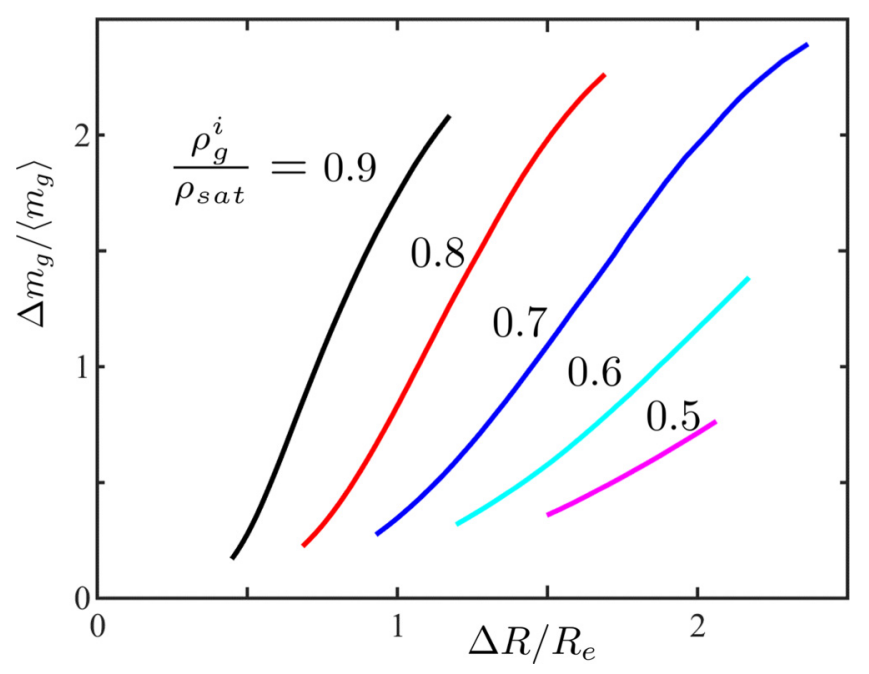

FIG. 4. Dimensionless oscillation amplitude of the mass of gas in the bubble vs dimensionless amplitude of the radial oscillations for different values of the liquid undersaturation $\rho_{g}^{i} / \rho_{\text {sat }}$ as in Fig. 1. The pressure amplitude increases from the lower left to the upper right, surface tension effects not included.

A cross-plot of the quantities of the previous two figures is provided in Fig. 4, in which each line is the dimensionless amplitude of the mass oscillations, $\Delta m_{g} /\left\langle m_{g}\right\rangle$, vs the nondimensional radius excursion $\Delta R / R_{e}$, for fixed $\rho_{g}^{i} / \rho_{\text {sat }}$. The range of the curves is the same as in the previous three figures, with $P_{*}$ increasing and the frequency decreasing as the lines are traversed from the lower left to the upper right. Again we see that, at the higher frequencies, the amplitude of the mass oscillations is small while that of the radial oscillations remains substantial. As the frequency decreases and the pressure amplitude increases, the amplitude of the mass oscillations increases substantially, more as the gas content of the liquid becomes greater. In considering these results it should be kept in mind that, unless $\Lambda$ is very small, the first and last terms in the bubble mass equation (13) tend to lose accuracy as $\Delta R / R$ approaches or exceeds 1, as explained in Sec. II. A more precise calculation should include the effect of convection, which would preclude the possibility of an analytic solution comparable to (13) and, therefore, the explicit identification of history effects.

Figure 5 is a graph of $\langle R\rangle / R_{e}$ vs $R_{e} / \sqrt{\pi D / \omega}$. The dependence on frequency tends to become weaker as frequency increases because, with the decreasing amplitude of mass fluxes, relatively small-amplitude oscillations are sufficient to provide the area changes necessary to preserve the mean bubble mass so that a small amount of nonlinearity (which is responsible for the difference between $\langle R\rangle$ and $R_{e}$ ) is sufficient. This regime is achieved later, of course, as the undersaturation increases and $\rho_{g}^{i} / \rho_{\text {sat }}^{0}$ becomes smaller. The fact that $\langle R\rangle / R_{e}>1$ in this parameter range suggests that the nature of the oscillations is such that the bubble radius is larger than $R_{e}$ during a substantial fraction of the oscillation period. The trend is reversed at low frequencies, where $\langle R\rangle / R_{e}<1$. Here the compressive phase is longer than the expansion phase, but the mass gained during the latter balances that lost during the former. These considerations are illustrated in Fig. 6 in which $R / R_{e}$ is plotted over a complete cycle for several values of $\rho_{g}^{i} / \rho_{\text {sat }}, R_{e} / \sqrt{\pi D / \omega}$ and $P_{*}$. The solid line, for $R_{e} / \sqrt{\pi D / \omega}=0.065, \rho_{g}^{i} / \rho_{\text {sat }}=0.8$, and $P_{*}=0.858$, is typical of the radius response at low frequency. One observes a marked asymmetry with respect to the maximum, with a compression phase much longer than the expansion phase due to the slowness of mass diffusion out of the bubble. On Fig. 5 this situation corresponds to the decreasing part of the curves on the left. The asymmetry is much less marked for the larger frequency examples for $R_{e} / \sqrt{\pi D / \omega}=0.36$. The dashed line is for $\rho_{g}^{i} / \rho_{\text {sat }}=0.8$ and $P_{*}=0.74$ and the dash-dotted line is for $\rho_{g}^{i} / \rho_{\text {sat }}=0.5$ and $P_{*}=0.93$. The liquid is very undersaturated in the last case and the amplitude necessary to ensure threshold conditions 


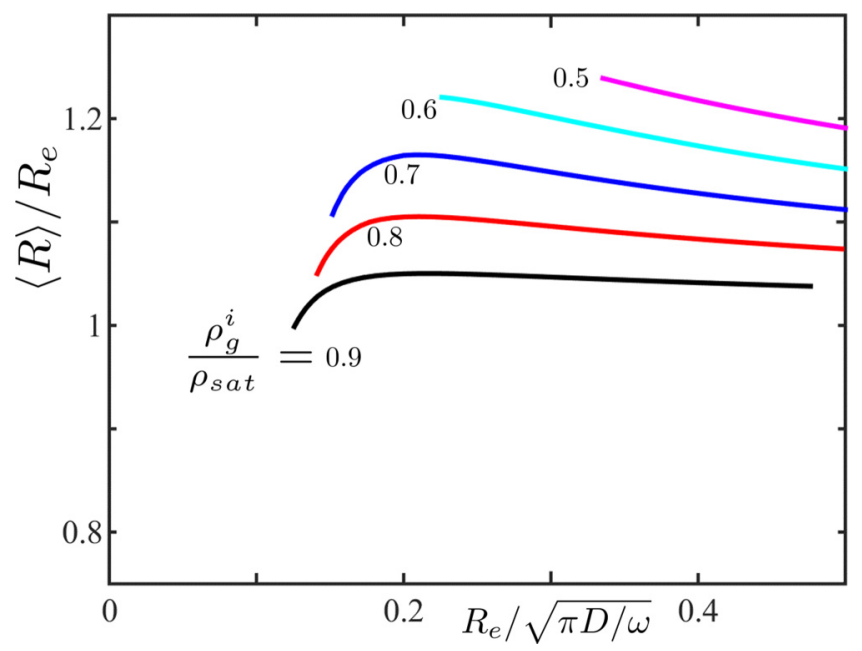

FIG. 5. Dimensionless mean bubble radius over a complete oscillation period vs the dimensionless bubble radius $R_{e} / \sqrt{\pi D / \omega}$ for different liquid undersaturations as in Fig. 1, surface tension effects not included.

is correspondingly larger. These curves are strikingly different from those found when the bubble inertia is important, in which case the expansion phase is usually much longer than the contraction phase.

It is also of interest to consider the relative magnitude of the second and third terms in (13) in the course of an oscillation. A few examples are shown in Figs. 7(a) to 7(d). Here the solid line is the quasisteady term $\left(\rho_{g}^{i}-\rho_{g}^{s f}\right) / R$ while the dashed line is the memory integral; both terms are nondimensionalized by division by $\rho_{\mathrm{sat}} / R_{e}$. When the parameter $\Lambda=R_{e} / \sqrt{\pi D / \omega}$, which is essentially the Péclet number, is small [Figs. 7(a) and 7(b)], the history term is smaller than the

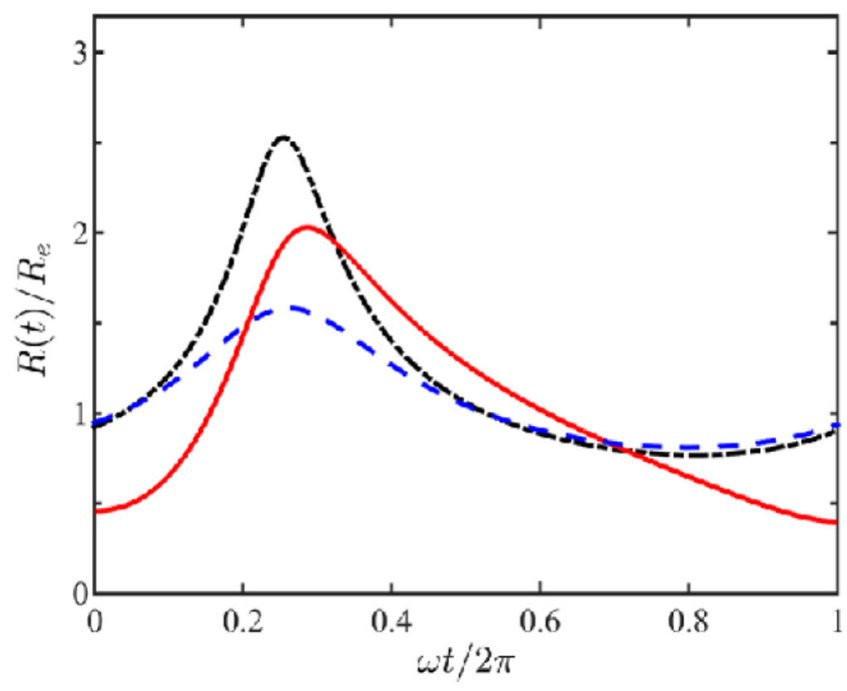

FIG. 6. Dimensionless bubble radius vs time over one complete period of the ambient pressure oscillations. Solid line: $R_{e} / \sqrt{\pi D / \omega}=0.065, \rho_{g}^{i} / \rho_{\text {sat }}=0.8$, and $P_{*}=0.86$; dashed line: $R_{e} / \sqrt{\pi D / \omega}=0.36, \rho_{g}^{i} / \rho_{\text {sat }}=$ 0.8 , and $P_{*}=0.74$; dash-dotted line: $R_{e} / \sqrt{\pi D / \omega}=0.36, \rho_{g}^{i} / \rho_{\text {sat }}=0.5$, and $P_{*}=0.93$. 

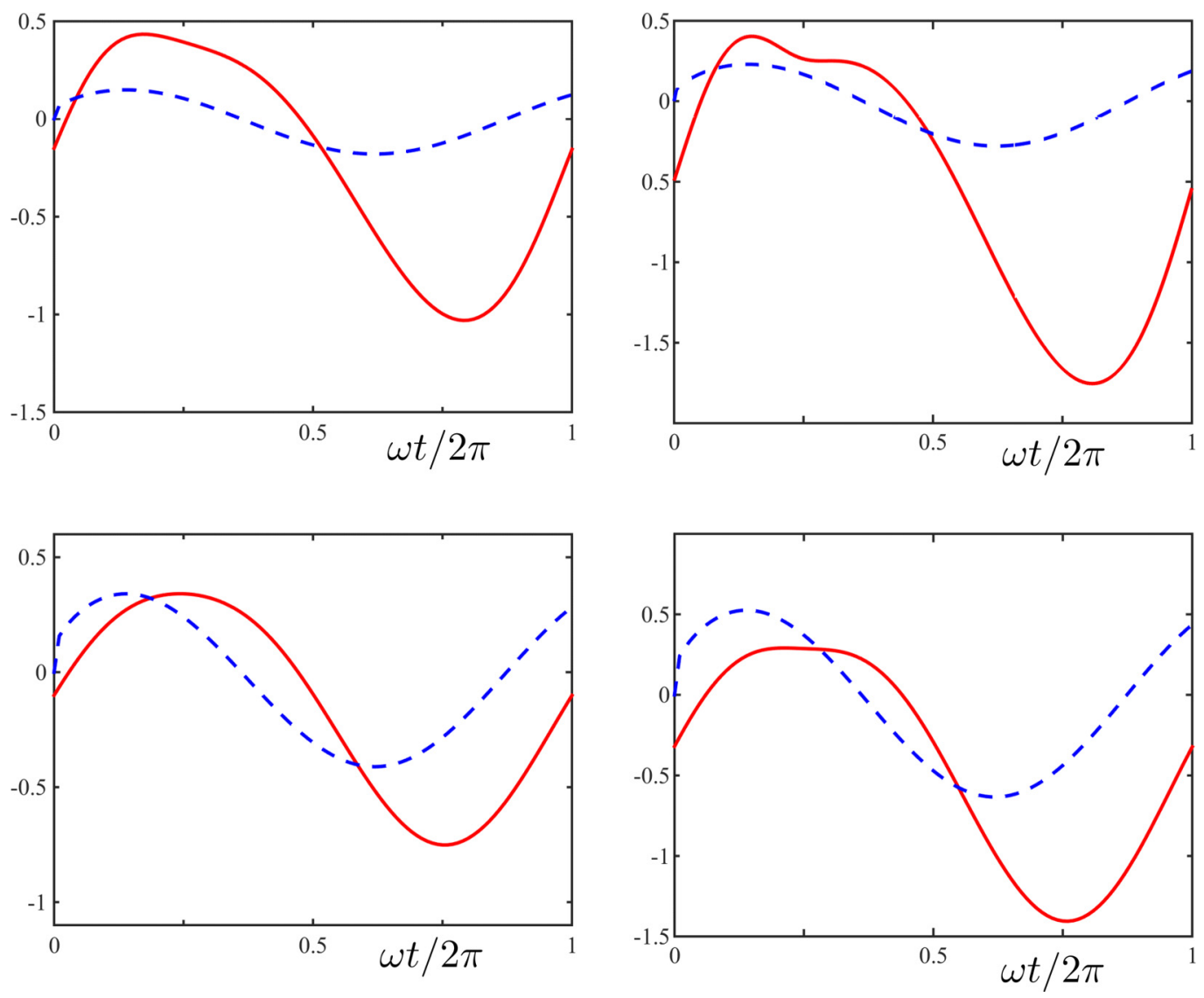

FIG. 7. The normalized quasisteady (second) term of (13) (solid line) and the history term in the course of one oscillation period at threshold conditions. The two panels in the first line are for $\Lambda=0.15, \Delta R / R=$ $0.95, \rho_{g}^{i} / \rho_{\text {sat }}=0.90, P_{*}=0.69$, and $\Lambda=0.17, \Delta R / R_{e}=2.0, \rho_{g}^{i} / \rho_{\text {sat }}=0.70, P_{*}=0.92$; those in the second line for $\Lambda=0.43, \Delta R / R_{e}=0.47, \rho_{g}^{i} / \rho_{\text {sat }}=0.90, P_{*}=0.54$, and $\Lambda=0.43, \Delta R / R_{e}=1.03, \rho_{g}^{i} / \rho_{\text {sat }}=0.70$, $P_{*}=0.82$.

quasisteady term, as expected. As $\Lambda$ increases [Figs. 7(c) and 7(d)], the relative magnitude of the history effect increases, again as expected. It was argued in Sec. II that the expression for the history effect given in (13) gradually loses accuracy as $\Delta R / R_{e}$ increases. However, it can be noted in Figs. 7(c) and 7(d), for the same values of $\Lambda$ but $\Delta R / R_{e}=0.47$ and 1.03 , that the relation between the two terms is comparable, which suggests a limited influence of the magnitude of $\Delta R / R_{e}$. The difference in the vertical scales is mostly due to the different liquid saturation as can be seen by comparing with the figures in the first line.

These results are strongly dependent on the history effect as shown by comparing Fig. 1, obtained from the full equation (21), with Fig. 8; this latter figure has been generated by omitting the history term so that $(25)$ is replaced by

$$
\int_{t_{*}}^{t_{*} 2 \pi / \omega} R_{*}\left[\rho_{g *}^{i}-\rho_{g *}^{s f}(t)\right] d t_{*}=0 .
$$

The lines are essentially horizontal, suggesting an independence of the threshold amplitude from frequency, except at very low frequency. This trend can be readily explained by recalling that at large frequencies the alternating mass exchange with the liquid has a negligible effect on the oscillation 


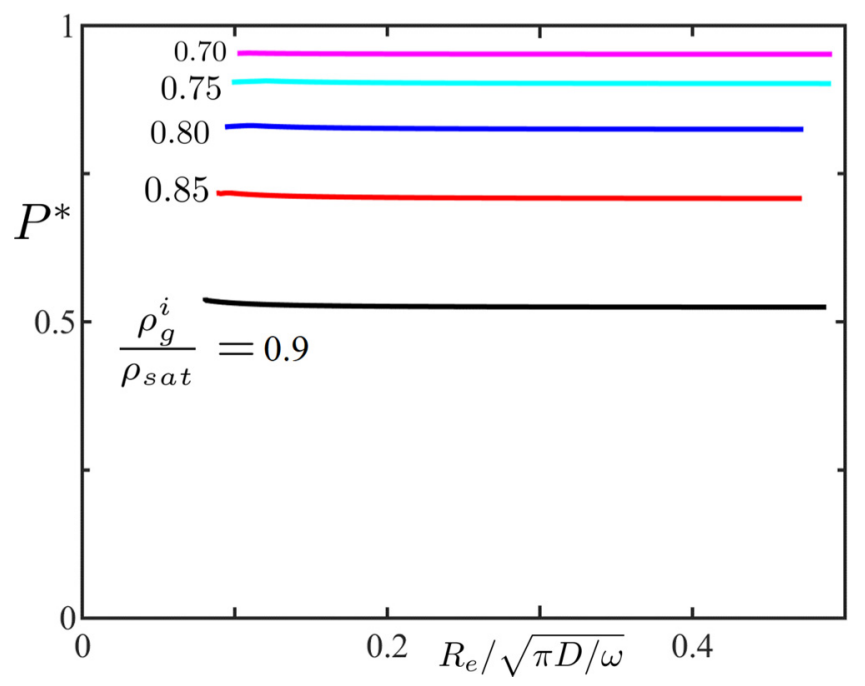

FIG. 8. Dimensionless rectified diffusion threshold amplitude $P_{*}$ vs dimensionless bubble size $R_{e} / \sqrt{\pi D / \omega}$ for different values of the liquid undersaturation $\rho_{g}^{i} / \rho_{\text {sat }}$ without history effects, surface tension not included.

amplitude. In these conditions, the area difference between contraction and expansion and, therefore, the net mass inflow into the bubble, only comes to depend on the pressure amplitude. A certain degree of undersaturation requires a corresponding net mass inflow and, therefore, a certain area change and a certain pressure amplitude. This argument can be given a quantitative form by noting that, in this limit, (31) can be approximated as

$$
\left[1-P_{*} \sin t_{*}\right] R_{*}^{3}\left(t_{*}\right) \simeq 1 .
$$

Thus, remembering also that $\rho_{g *}^{s f}=1-P_{*} \sin t_{*}$, (27) gives

$$
\rho_{g_{*}}^{i} \int_{0}^{2 \pi / \omega}\left[1-P_{*} \sin t_{*}\right]^{-1 / 3} d t_{*}=\int_{0}^{2 \pi / \omega}\left[1-P_{*} \sin t_{*}\right]^{2 / 3} d t_{*},
$$

which can be solved to give $P_{*}$ vs $\rho_{g *}^{i}$ independently of frequency. This relation is graphed in Fig. 9 . This exclusive dependence on pressure amplitude becomes less accurate as the mass fluxes become larger with decreasing frequency.

We now turn to a consideration of the effects of surface tension, the most important of which-of increasing importance as the bubble radius becomes smaller-is to inhibit the radial expansion. Thus it may be expected that, as a consequence, the threshold for rectified diffusion will be generally larger and exhibit a much stronger dependence on the radius than that shown in Fig. 1. This expectation is borne out by Fig. 10, which is comparable to Fig. 1 except for the fact that the parameter $\beta$, defined in (24), equals 0.1 , rather than 0 , and that the liquid gas content needs to be significantly increased to allow for a threshold in the presence of the strong surface-tension-induced overpressure. To give an idea of the orders of magnitude of actual physical quantities we may note that, for water and air, with $D=2 \times 10^{-9} \mathrm{~m}^{2} / \mathrm{s}$ and $\sigma=0.07 \mathrm{~N} / \mathrm{m}, \beta=0.1$ corresponds to a frequency

$$
\frac{\omega}{2 \pi}=\frac{1}{2} D\left(\frac{\beta p_{0}^{\infty}}{2 \sigma}\right)^{2} \simeq 10 \mathrm{~Hz}
$$

while $R_{e} / \sqrt{\pi D / \omega} \sim 0.3$, for example, would correspond to $R_{e} \sim 30 \mu \mathrm{m}$. It is not surprising to see such a strong effect of surface tension for this small radius. 


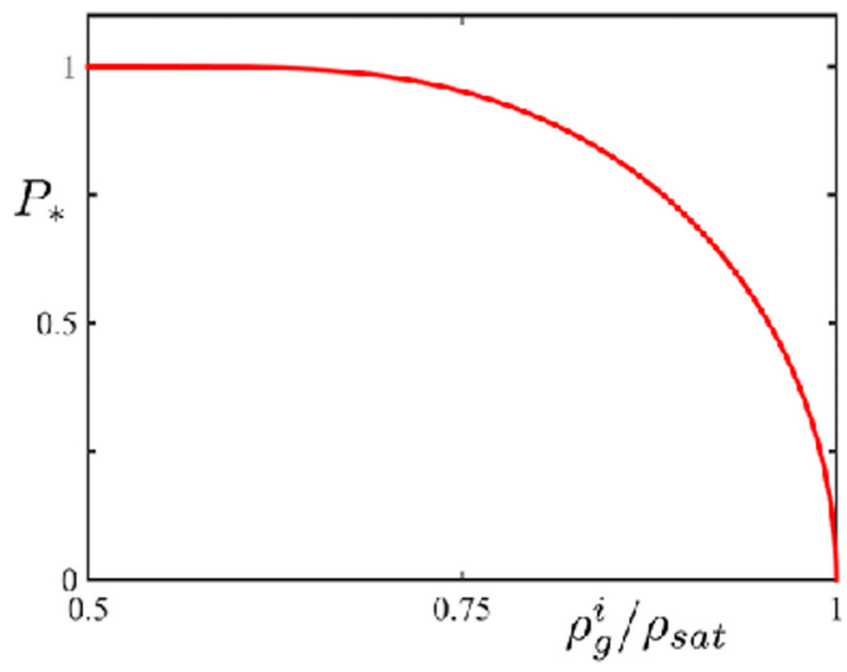

FIG. 9. Dimensionless rectified diffusion threshold as a function of the liquid undersaturation $\rho_{g}^{i} / \rho_{\text {sat }}$ as given in (27) in the high-frequency limit without history effects or surface tension.

A direct comparison of the threshold with and without surface tension, $\beta=0.01$ and $\beta=0$, is given in Fig. 11 for $\rho_{g}^{i} / \rho_{\text {sat }}=0.9$. The smaller value of $\beta$ as compared with Fig. 10 causes smaller differences which, however, remain significant.

\section{MASS TRANSFER IN A CONSTANT PRESSURE FIELD}

We now turn to the case of a constant pressure field $p_{\infty}$, the original situation studied in Ref. [5]. Now it is convenient to use the initial bubble radius $R(0)$ as scale and we set $R_{*}(t)=R(t) / R(0)$.

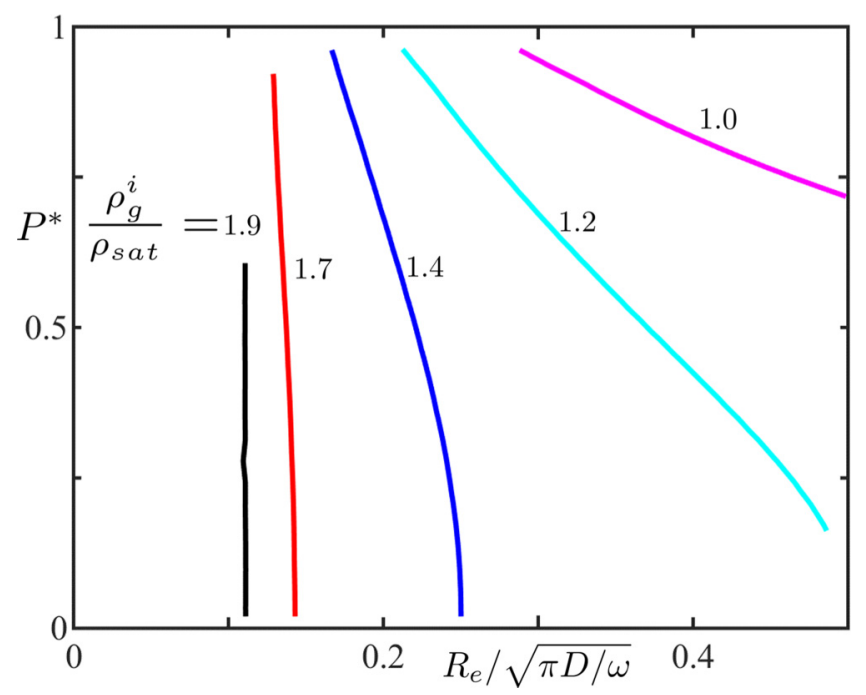

FIG. 10. Dimensionless rectified diffusion threshold amplitude $P_{*}$ vs dimensionless bubble size $R_{e} / \sqrt{\pi D / \omega}$ for different values of the liquid undersaturation $\rho_{g}^{i} / \rho_{\text {sat }}$ with surface tension effects included. The parameter $\beta=2 \sigma /\left(p_{0}^{\infty} \sqrt{\pi D / \omega}\right)$ equals 0.1 . 


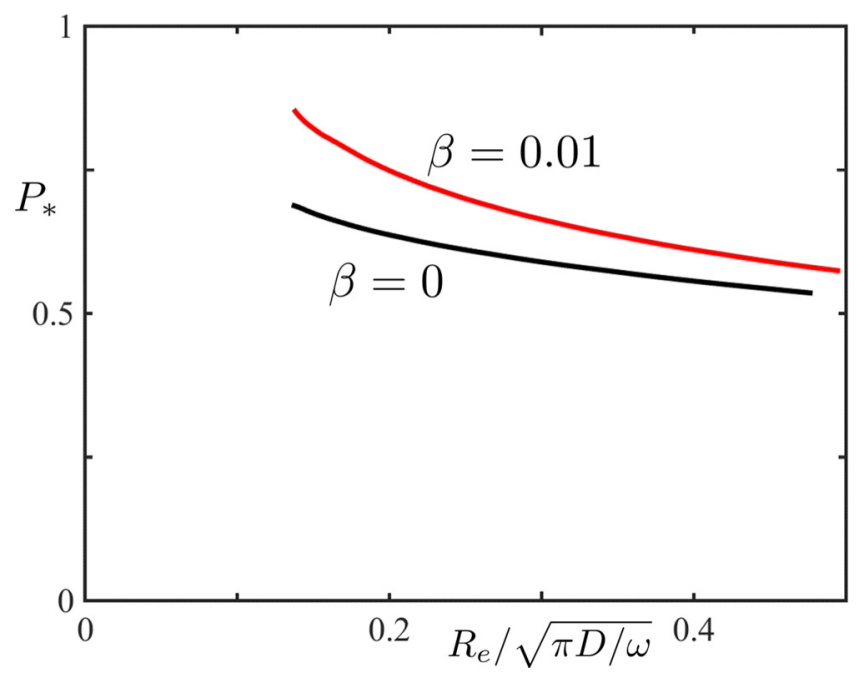

FIG. 11. Comparison of the dimensionless rectified diffusion threshold amplitude $P_{*}$ vs the dimensionless bubble size $R_{e} / \sqrt{\pi D / \omega}$ with and without surface tension effects for $\rho_{g}^{i} / \rho_{\text {sat }}=0.9$; the parameter $\beta$ is defined in (24).

The analog of (26) is now

$$
\frac{\left[1+\alpha / R_{*}(t)\right] R_{*}^{3}(t)}{1+\alpha}=\frac{m_{g}(t)}{m_{g}(0)},
$$

with $\alpha$ given by (24).

Figure 12 shows the time to complete dissolution as a function of the gas content of the liquid for three values of the parameter $\alpha, 1.42,0.142$, and 0.0142 , which, in water at $22^{\circ} \mathrm{C}$, would correspond to initial radii of 1,10 , and $100 \mu \mathrm{m}$. For dissolution to be possible it is necessary that $\rho_{g}^{i} / \rho_{\text {sat }}<1+\alpha$

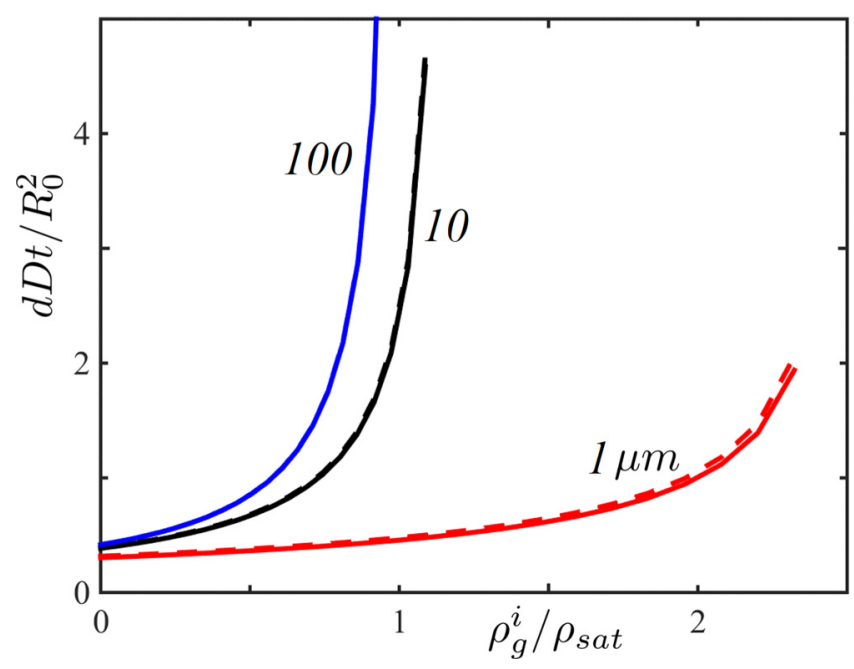

FIG. 12. Dimensionless time to complete dissolution of gas bubbles with different initial radii as a function of the gas content of the liquid; the ambient pressure is constant. The dashed lines show the results when the history term is omitted. 


\section{HISTORY EFFECTS ON THE GAS EXCHANGE BETWEEN A ...}

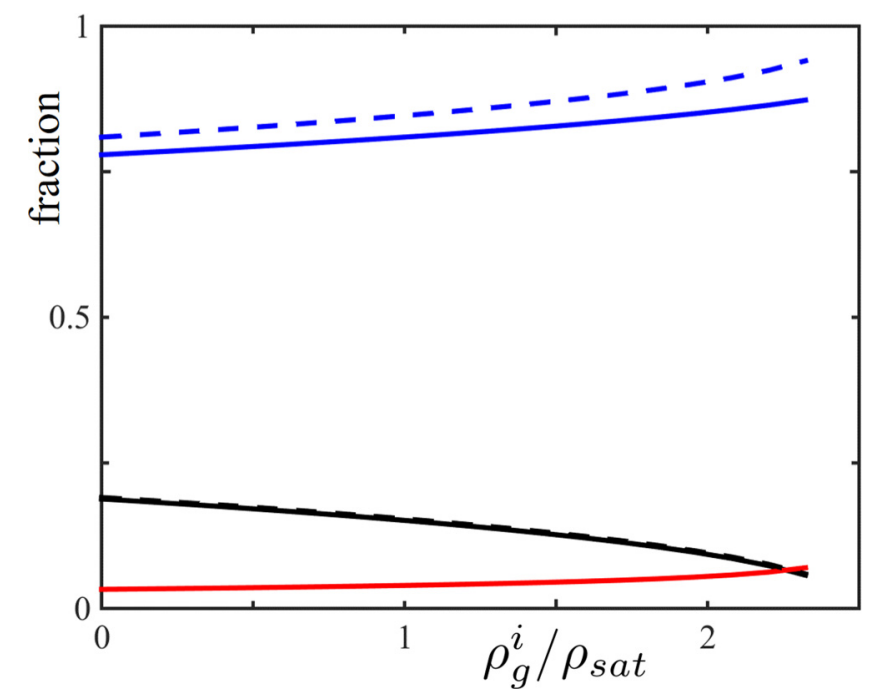

FIG. 13. Fractional contributions of the three terms in (13) to the dissolution time of an air bubble in water with an initial radius of $1 \mu \mathrm{m}$. In ascending order the lines show the contributions of the history term, of the term proportional to $t^{-1 / 2}$ and of the term inversely proportional to $R$. The dashed lines are the corresponding contributions in the absence of history effects.

and the dissolution becomes markedly slower as the limiting value is approached until it diverges when it is attained. The difference due to the removal of the history term (dashed line) is very small and only visible for the smallest bubble. In the other cases, the effect of the surface-tension overpressure is so small over the major fraction of the bubble lifetime that the two results with and without memory effects are indistinguishable within the thickness of the lines. It is somewhat surprising that the slight difference between the two theories is seen to increase with the degree of saturation, as one would expect that faster dissolution would increase the significance of the memory term. While this is true, the effect becomes secondary compared to the influence of the memory term over the longer bubble lifetime produced by a greater amount of dissolved gas. Even so, the effect remains small, mostly of the order of a fraction of a percent. Figure 13 shows the relative importance of the three terms in (13). Figure 14 illustrates the effect of the memory term on the time to growth up to 10 times the initial radius.

\section{A MULTICOMPONENT BUBBLE}

As a last example of a situation in which the boundary condition at the bubble surface is time dependent, we consider a bubble consisting of a mixture of two different gases, denoted by indices $a$ and $b$, each one with its own solubility and diffusivity. This problem has been considered in Ref. [11] in the limit in which one of the gases is insoluble and in Ref. [17] under the assumption of a constant dissolved gas mole fraction at the bubble surface; in neither work are history effects considered. The latter authors compared the results of a numerical simulation, including convection and the time dependence of the surface concentration and of the boundary, with their quasisteady approximate solution, finding a reasonable agreement between them. In this analysis we neglect surface tension and assume that the binary diffusivity in the gaseous phase is sufficiently rapid for the two constituents to remain fully mixed in the bubble interior.

The masses $m_{a}$ and $m_{b}$ of each component of the gas mixture are each determined by an equation of the form (13), after which the bubble radius follows from Dalton's law and the equation 


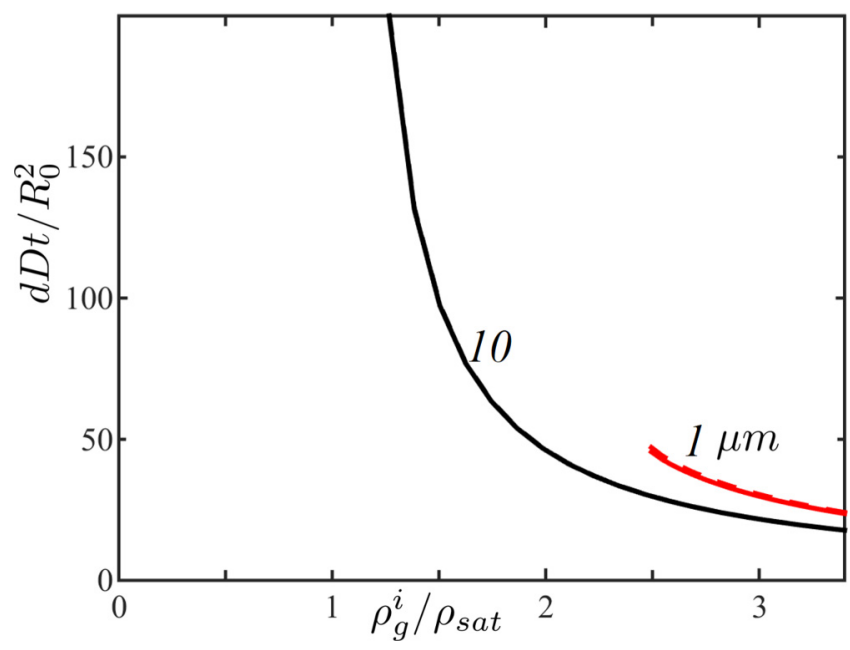

FIG. 14. Dimensionless time for growth up to 10 times the initial radius of an air bubble in water as a function of the liquid supersaturation; the initial bubble radius is $1 \mu \mathrm{m}$. The dashed line is the result in the absence of the history term in (13).

of state (10) as

$$
\left(\frac{4}{3} \pi R^{3}\right)\left(p_{a}+p_{b}\right)=\left(\frac{4}{3} \pi R^{3}\right) p_{\infty}=\left(\frac{m_{a}}{M_{a}}+\frac{m_{b}}{M_{b}}\right) R_{G} T .
$$

For a numerical example we consider the case of a bubble consisting of a mixture of nitrogen (component $a$ ) and $\mathrm{CO}_{2}$ in water at $20^{\circ} \mathrm{C}$ under a constant $101.3 \mathrm{kPa}$ ambient pressure; at the initial instant there are no gases dissolved in the water. The values of the parameter $d$ defined in (3) are $d_{a}=0.04$ and $d_{b}=0.80$, respectively, and the respective diffusivities $D_{a}=1.88 \times 10^{-9} \mathrm{~m}^{2} / \mathrm{s}$ and $D_{b}=1.92 \times 10^{-9} \mathrm{~m}^{2} / \mathrm{s}$. On the basis of the time scale for the dissolution of a monocomponent bubble, it was shown in Sec. II that neglect of convection was legitimate for small $d$. For the present case of a multicomponent bubble it is the less soluble gas which determines the dissolution time, and therefore the fact that $d_{b}$ is not small does not introduce a significant error. Figure 15 shows the

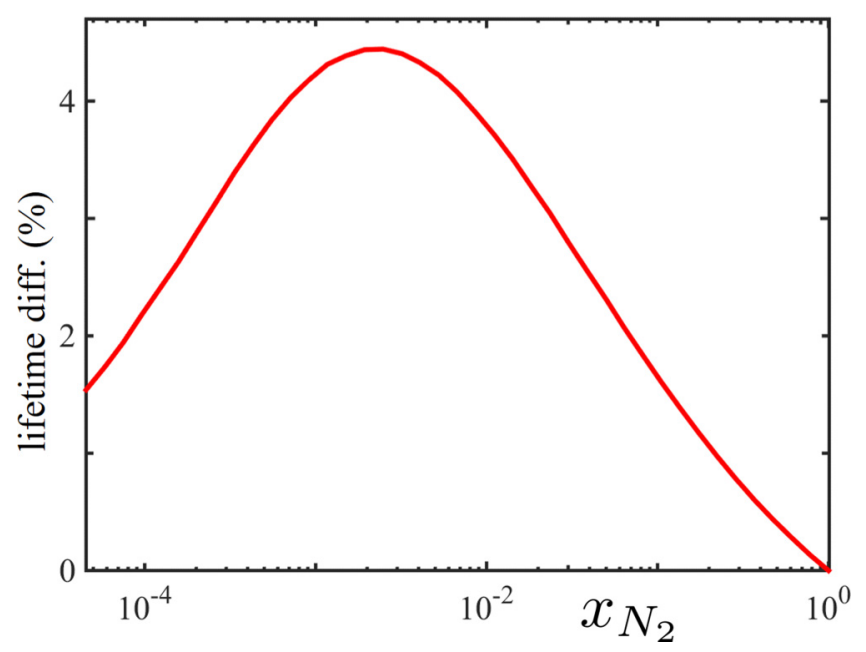

FIG. 15. Percent difference between the lifetimes of a nitrogen- $\mathrm{CO}_{2}$ bubble with and without history effects vs the initial nitrogen mole fraction in the bubble (note the logarithmic scale). 


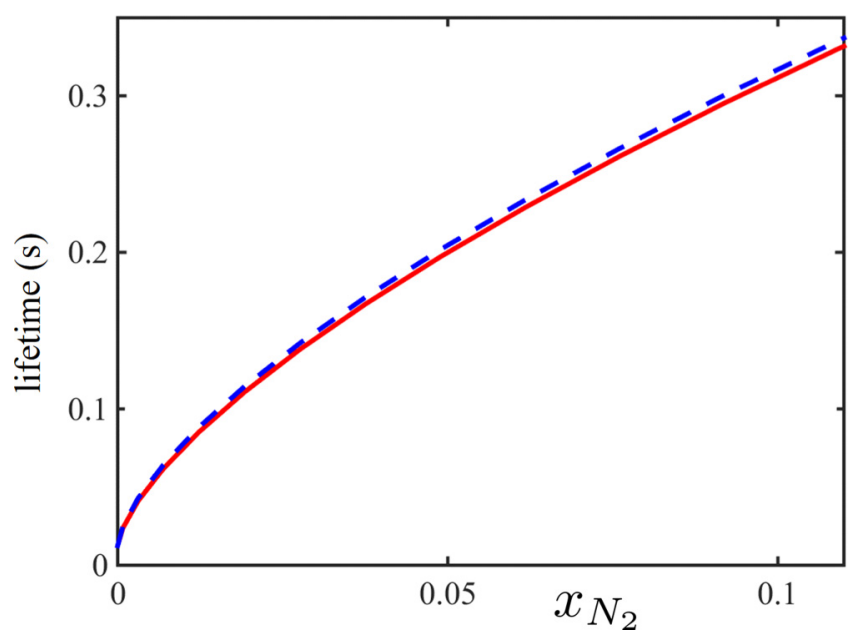

FIG. 16. Comparison of the lifetimes of a nitrogen- $\mathrm{CO}_{2}$ bubble with (solid line) and without history effects vs the initial nitrogen mole fraction.

percent difference between the predicted lifetimes with and without the history effect as a function of the nitrogen mole fraction $x_{N_{2}}$ initially present in the bubble. A maximum of about $4 \%$ is found for $x_{N_{2}} \simeq 2 \times 10^{-3}$. Figure 16, in which the solid line includes the history effect, is a comparison of the actual lifetimes in the range of small $x_{N_{2}}$. The more significant differences are limited to the range of small $x_{N_{2}}$ because $\mathrm{CO}_{2}$ dissolves much faster than nitrogen. Thus, when the nitrogen amount is not small, the $\mathrm{CO}_{2}$ dissolves very quickly and the situation becomes similar to that of a single-component nitrogen bubble except for a very short initial period.

\section{SUMMARY}

Henry's law dictates the concentration of dissolved gas at the surface of a gas bubble in a liquid. When the bubble internal pressure changes, either in response to a varying ambient pressure, because of surface tension in the presence of radius changes, or for other causes, the dissolved gas concentration becomes time dependent and the history effects characteristic of all diffusive processes appear. In order to focus on these effects, we have considered the limit in which the diffusive layer is much thicker than the bubble radius and the frequency of the pressure variation is much slower than the bubble resonance frequency. Not surprisingly we have found that when the dissolution or growth take place under a constant ambient pressure, so that the sole agent of the internal pressure variation is the changing radius, history effects are small. In the last example considered, in which the bubble consists of the mixture of two gases, history effects can be somewhat larger but they amount to a few percent at most.

When the internal pressure changes in response to variations of the ambient pressure, however, the history effect on rectified diffusion can be more important. In this case, the different frequency responses of the gas inflow or outflow and surface area increase or decrease during the pressure cycle are important. Since the former is a relatively slow process, at low frequency there are large mass exchanges with the liquid which require large expansions and contractions of the bubble. At higher frequency, changes in the surface area become the dominant effect and the oscillation amplitude is smaller.

\section{ACKNOWLEDGMENTS}

The authors are grateful to an anonymous referee whose perceptive comments prompted the analysis given in Sec. III. This study was made possible by a grant from the Gulf of Mexico 
Research Initiative. Data are publicly available through the Gulf of Mexico Research Initiative Information and Data Cooperative (GRIIDC) at DOI 10.7266/N77M05ZD. The funders had no role in study design, data collection and analysis, decision to publish, or preparation of the manuscript.

\section{APPENDIX: AN ESTIMATE OF THE ERROR IN (12)}

In the convection-diffusion equation let

$$
x=\frac{r}{R}, \quad u(x, t)=x\left(\rho_{g}-\rho_{g}^{i}\right),
$$

to find

$$
\frac{\partial u}{\partial t}+\frac{\dot{R}}{R}\left(\frac{1}{x^{3}}-1\right)\left(x \frac{\partial u}{\partial x}-u\right)=\frac{D}{R^{2}} \frac{\partial^{2} u}{\partial x^{2}} .
$$

The second term in the left-hand side can be estimated as before and neglected at low frequency. In terms of the new time variable

$$
\tau=\int_{0}^{t} \frac{d t^{\prime}}{R^{2}\left(t^{\prime}\right)}
$$

the equation then becomes (approximately)

$$
\frac{\partial u}{\partial \tau}=D \frac{\partial^{2} u}{\partial x^{2}} .
$$

Upon taking the Laplace transform and solving with the appropriate boundary condition we find

$$
\tilde{u}(x, s)=\tilde{u}(x=1, s) e^{-\sqrt{s / D}(x-1)},
$$

from which

$$
\frac{1}{x}\left(\frac{\partial \tilde{u}}{\partial x}-\frac{\tilde{u}}{x}\right)=-\frac{s \tilde{u}(x=1, s) e^{-\sqrt{s / D}(x-1)}}{x \sqrt{s D}}-\frac{\tilde{u}(x=1, s) e^{-\sqrt{s / D}(x-1)}}{x^{2}} .
$$

Upon inverting the transform we find

$$
\begin{aligned}
\frac{1}{x}\left(\frac{\partial u}{\partial x}-\frac{u}{x}\right)= & -\frac{1}{x} \int_{0}^{\tau} \frac{d u(x=1, \theta)}{d \theta} \frac{e^{(x-1)^{2} / 4 D(\tau-\theta)}}{\sqrt{\pi(\tau-\theta)}} d \theta \\
& -\frac{1}{x} \int_{0}^{\tau} u(x=1, \tau-\theta) \frac{(x-1) e^{(x-1)^{2} / 4 D \theta}}{2 \sqrt{\pi D \theta^{3}}} d \theta
\end{aligned}
$$

We are interested in the value of this quantity at $x=1$. For the first term we recall the boundary condition (7) to find

$$
\frac{d u(x=1, \theta)}{d \theta}=\delta(\theta)\left[\rho_{g}^{s f}(0)-\rho_{g}^{i}\right]+H(\theta) \frac{\partial \rho_{g}^{s f}}{d \theta}
$$

from which

$$
\left[\frac{1}{x} \int_{0}^{\tau} \frac{d u(x=1, \theta)}{d \theta} e^{(x-1)^{2} / 4 D(\tau-\theta)} \frac{d \theta}{\sqrt{\pi(\tau-\theta)}}\right]_{x=1}=-\frac{\rho_{g}^{s f}(0)-\rho_{g}^{i}}{\sqrt{\pi D \tau}}-\int_{0}^{\tau} \frac{d \rho_{g}^{s f}}{d \theta} \frac{d \theta}{\sqrt{\pi D(\tau-\theta)}} .
$$


For the second term we set

$$
\eta=\frac{x-1}{2 \sqrt{D(\tau-\theta)}}
$$

to find

$$
\begin{aligned}
& \frac{1}{x} \int_{0}^{\tau} u(x=1, \tau-\theta) \frac{x-1}{2 \sqrt{\pi D \theta^{3}}} e^{(x-1)^{2} / 4 D \theta} d \theta \\
& \quad=\frac{1}{x \sqrt{\pi}} \int_{(x-1) / 2 \sqrt{D \tau}}^{\infty} u\left(x=1, \tau-\frac{(x-1)^{2}}{4 D \eta^{2}}\right) e^{-\eta^{2}} d \eta
\end{aligned}
$$

Upon evaluating at $x=1$ this is simply

$$
\left[\frac{1}{x} \int_{0}^{\tau} u(x=1, \tau-\theta) \frac{x-1}{2 \sqrt{\pi D \theta^{3}}} e^{(x-1)^{2} / 4 D \theta} d \theta\right]_{x=1} d \theta=u(x=1, \tau)=\rho_{g}^{s f}(\tau)-\rho_{g}^{i} .
$$

In conclusion we have found

$$
\begin{aligned}
{\left[\frac{\partial \rho_{g}}{\partial r}\right]_{r=R} } & =\frac{1}{R}\left[\frac{1}{x} \frac{\partial u}{\partial x}-\frac{u}{x^{2}}\right]_{x=1} \\
& =-\frac{1}{R}\left[\frac{\rho_{g}^{s f}(0)-\rho_{g}^{i}}{\sqrt{\pi D \tau}}+\rho_{g}^{s f}(\tau)-\rho_{g}^{i}+\int_{0}^{\tau} \frac{d \rho_{g}^{s f}}{d \theta} \frac{d \theta}{\sqrt{\pi D(\tau-\theta)}}\right],
\end{aligned}
$$

which differs from the earlier results only by the substitution of the original time variable $t$ by the modified time variable $\tau$.

We note that, by the first mean value theorem, for some $t_{m}$ with $0 \leqslant t_{m} \leqslant t$,

$$
R \sqrt{\tau}=R(t) \sqrt{\int_{0}^{t} \frac{d t^{\prime}}{R^{2}\left(t^{\prime}\right)}}=R(t) \sqrt{\frac{t}{R^{2}\left(t_{m}\right)}} .
$$

If the radius is constant, this is just $\sqrt{t}$ and (13) is recovered. The radius of a dissolving bubble in a constant pressure field decreases very slowly for a dominant fraction of the bubble lifetime. In this case, therefore, the difference between using $t$ or $\tau$ will not be large. For an oscillating bubble with a radius varying between $R_{e}-\Delta R$ and $R_{e}+\Delta R$, identifying (A14) with $\sqrt{t}$ introduces an error bounded by $\left(1-\Delta R / R_{e}\right) /\left(1+\Delta R / R_{e}\right)$ and $\left(1+\Delta R / R_{e}\right) /\left(1-\Delta R / R_{e}\right)$, which will be small provided $\Delta R / R_{e}$ is small.

[1] A. Eller and H. G. Flynn, Rectified diffusion during nonlinear pulsations of cavitation bubbles, J. Acoust. Soc. Am. 37, 493 (1965).

[2] L. A. Crum, Measurement of the growth of air bubbles by rectified diffusion, J. Acoust. Soc. Am. 68, 203 (1980).

[3] M. P. Brenner, S. Hilgenfeldt, and D. Lohse, Single-bubble sonoluminescence, Rev. Mod. Phys. 74, 425 (2002).

[4] M. M. Fyrillas and A. J. Szeri, Dissolution or growth of soluble spherical oscillating bubbles, J. Fluid Mech. 277, 381 (1994).

[5] P. S. Epstein and M. S. Plesset, On the stability of gas bubbles in liquid-gas solutions, J. Chem. Phys. 18, 1505 (1950).

[6] P. B. Duncan and D. Needham, Test of the Epstein-Plesset model for gas microparticle dissolution in aqueous media: Effect of surface tension and gas undersaturation in solution, Langmuir 20, 2567 (2004). 
[7] P. B. Duncan and D. Needham, Microdroplet dissolution into a second-phase solvent using a micropipet technique: Test of the Epstein-Plesset model for an aniline-water system, Langmuir 22, 4190 (2006).

[8] D. Lohse and X. Zhang, Surface nanobubbles and nanodroplets, Rev. Mod. Phys. 87, 981 (2015).

[9] P. Peñas-López, M. A. Parrales, J. Rodríguez-Rodríguez, and D. van der Meer, The history effect in bubble growth and dissolution, part 1: Theory, J. Fluid Mech. 800, 180 (2016).

[10] S. Chu and A. Prosperetti, Dissolution and growth of a multicomponent drop in an immiscible liquid, J. Fluid Mech. 798, 787 (2016).

[11] M. C. Weinberg, P. I. K. Onorato, and D. R. Uhlmann, Behavior of bubbles in glassmelts, II: Dissolution of a stationary bubble containing a diffusing and non-diffusing gas, J. Am. Ceram. Soc. 63, 435 (1980).

[12] M. C. Weinberg, P. I. K. Onorato, and D. R. Uhlmann, Behavior of bubbles in glassmelts, I: Dissolution of a stationary bubble containing a single gas, J. Am. Ceram. Soc. 63, 175 (1980).

[13] R. S. Subramaniam and M. C. Weinberg, The role of convective transport in the dissolution or growth of a gas bubble, J. Chem. Phys. 72, 6811 (1980).

[14] L. N. Tao, Dynamics of growth or dissolution of a gas bubble, J. Chem. Phys. 69, 4189 (1978).

[15] L. N. Tao, The general solution and its analyticity for growth or dissolution of a gas bubble, J. Chem. Phys. 71, 3455 (1979).

[16] Y. A. Iliinski, P. S. Wilson, and M. F. Hamilton, Bubble growth by rectified diffusion at high gas supersaturation levels, J. Acoust. Soc. Am. 124, 1950 (2008).

[17] M. C. Weinberg and S. Subramaniam, Dissolution of multi-component bubbles, J. Am. Ceram. Soc. 63, 527 (1980). 